Case

Report

\title{
Partial Anomalous Pulmonary Venous Connection Associated with Lung Cancer in the Same Lobe: Report of a Case
}

\author{
Keisuke Asakura, MD, ${ }^{1}$ Yotaro Izumi, MD,${ }^{1}$ Mitsutomo Kohno, MD, ${ }^{1}$ \\ Masazumi Watanabe, MD, ${ }^{1}$ Takahide Arai, MD, ${ }^{2}$ and Hiroaki Nomori, MD ${ }^{1}$
}

\begin{abstract}
A 64-year-old man with primary lung cancer (cT1aN0M0) was diagnosed as having partial anomalous pulmonary venous connection (PAPVC) in the same lobe by preoperative chest computed tomography (CT). The anomalous vein originated from left upper lobe pulmonary vein and flowed into the left brachiocephalic vein. Although the patient was asymptomatic, cardiac catheterization revealed that pulmonary-systemic blood flow ratio $(\mathrm{Qp} / \mathrm{Qs}$ ratio) was $\mathbf{2 . 0}$, and his pulmonary arterial pressure was marginally elevated $(60 / 18 \mathrm{mmHg})$. We performed left upper lobectomy as the definitive treatment for both lung cancer and PAPVC. His pulmonary arterial pressure decreased after lobectomy $(33 / 16 \mathbf{~ m m H g})$. He is living well without relapse of lung cancer 56 months after surgery. Although PAPVC is detectable on computed tomography, out of 7 previous reports of PAPVC associated with lung cancer, only 2 cases were diagnosed preoperatively. The presence of PAPVC should be kept in mind before major lung resections.
\end{abstract}

Keywords: lung cancer, partial anomalous pulmonary venous connection

\section{Introduction}

Partial anomalous pulmonary venous connection (PAPVC) is a relatively uncommon congenital anomaly, with a reported incidence of $0.4 \%-0.7 \%$ in autopsy series of patients without known congenital heart disease. ${ }^{1,2)} \mathrm{In}$ this anomaly, blood from the anomalously drained portion of the lung is recirculated to the right side of the heart, creating a left to right shunt. To our knowledge, there are 7 reported cases of PAPVC associated with lung

${ }^{1}$ Division of General Thoracic Surgery, Department of Surgery, School of Medicine, Keio University, Tokyo, Japan

${ }^{2}$ Department of Cardiology, School of Medicine, Keio University, Tokyo, Japan

Received: March 13, 2012; Accepted: August 16, 2012

Corresponding author: Yotaro Izumi, MD. Division of General Thoracic Surgery, Department of Surgery, School of Medicine, Keio University, 35 Shinanomachi, Shinjuku-ku, Tokyo 160-8582, Japan

Email: yotaro2008@gmail.com

(C)2014 The Editorial Committee of Annals of Thoracic and Cardiovascular Surgery. All rights reserved. cancer. In these reports, preoperative diagnosis of PAPVC was obtained in only 2 cases. Here we report a case of left PAPVC which was diagnosed during the preoperative examinations for primary lung cancer.

\section{Case Report}

A 64-year-old man was referred to the department of cardiology with hypertension. Further examinations revealed that he had atrial fibrillation and mitral regurgitation, but he was followed-up. During follow-up, he was found to have a nodular ground-glass opacity with a maximal diameter of $2 \mathrm{~cm}$ in the left upper lobe on screening chest computed tomography (CT) (Fig. 1A). The shadow was diagnosed as lung adenocarcinoma by CT-guided percutaneous needle biopsy. Clinical stage was IA (T1aNOM0), and he was admitted to our department for treatment. Preoperative chest CT showed an anomalous blood vessel beside aortic arch (Fig. 1B). PAPVC was suspected and cardiac catheterization was done. Angiography showed that PAPVC originated from 


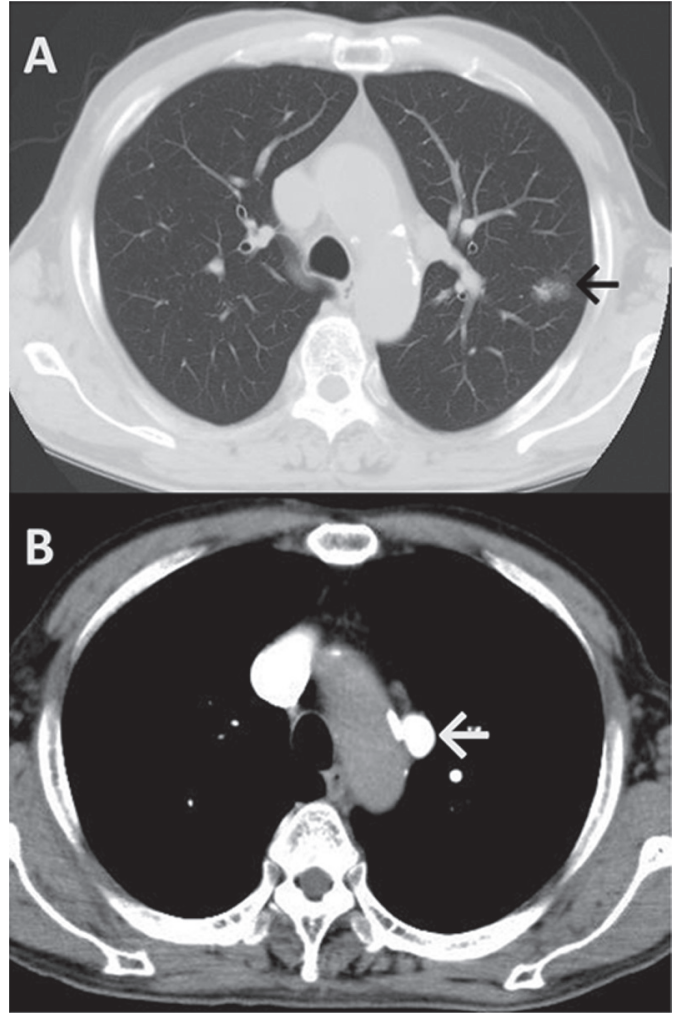

Fig. 1 (A) Chest computed tomography (CT) showing a nodular ground-glass opacity in left upper lobe (arrow). (B) Chest CT showing an anomalous vein beside aortic arch (arrow).

left upper lobe pulmonary vein and flowed into the left brachiocephalic vein (Fig. 2). The left upper pulmonary vein was located cranially from the hilum. Step-up of oxygen saturation in the left brachiocephalic vein (68\% in the right brachiocephalic vein, and $90 \%$ in the brachiocephalic vein) was observed with moderate pulmonary hypertention $(60 / 18 \mathrm{mmHg}$ measured by catheter, and 57/18 mmHg calculated on echocardiography). Venous blood from the left upper lobe flowed completely into the anomalous vein. Pulmonary-systemic blood flow ratio (Qp/Qs ratio) obtained by the Fick method during cardiac catheterization was 2.0. Echocardiography showed moderate mitral regurgitation, left ventricular dilation, and hypokinesis. Ejection Fraction (EF) was 53\%. Although PAPVC has been reported to be commonly associated with atrial level shunt, he had no other congenital heart anomalies, and his atrial fibrillation and mitral regurgitation were not considered to be associated with PAPVC. Preoperative blood gas analysis revealed partial arterial pressure of oxygen $(\mathrm{PaO} 2) 78 \mathrm{mmHg}$, and carbon dioxide (PaCO2) $49 \mathrm{mmHg}$ in room air. Spirometry revealed

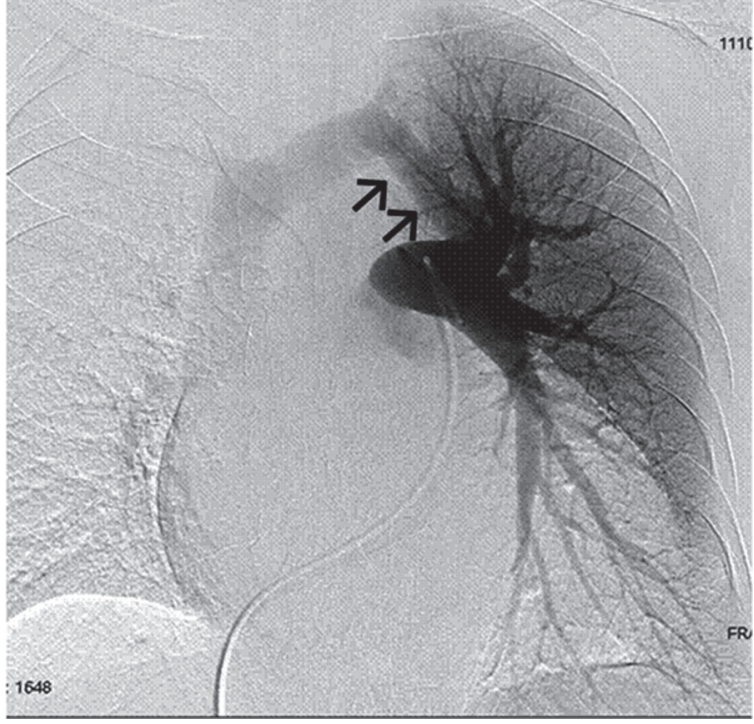

Fig. 2 Cardiac angiography revealed a major left-to-right shunt through an anomalous pulmonary vein (arrow) connecting to the left brachiocephalic vein.

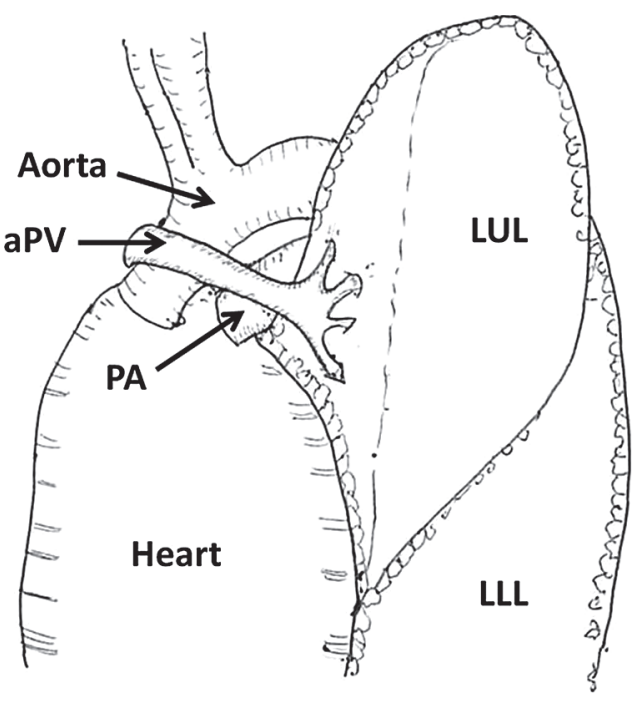

Fig. 3 Intraoperative schema of the anomalous vein. (PA: pulmonary artery; aPV: anomalous pulmonary vein; LUL: left upper lobe; LLL: left lower lobe)

normal respiratory functions. We assessed that left upper lobectomy was feasible because it was a definitive operation not only for primay lung cancer but also for PAPVC.

Operation was performed through a left anteriolateralthoracotomy approach under one-lung ventilation. The anomalous vein originated from the root of left upper pulmonary vein, traversed aortic arch and connected to the left brachiocephalic vein. Figure 3 shows the intraoperative schema of the anomalous vein. Left upper lobectomy 
Table 1 Reported cases of PAPVC associated with lung cancer

\begin{tabular}{lclccc}
\hline Author & Age/Gender & Location of PAPVC & Procedure & $\begin{array}{c}\text { Vascular } \\
\text { reconstruction }\end{array}$ & $\begin{array}{c}\text { Preoperative } \\
\text { diagnosis }\end{array}$ \\
\hline Black & $72 / \mathrm{F}$ & LUL to BCV & RPx & - & - \\
Takamori & $68 / \mathrm{M}$ & LUL to BCV & LULx & - & - \\
Takei & $58 / \mathrm{F}$ & LUL to BCV & LLLx & + & - \\
Miwa & $79 / \mathrm{F}$ & LUL to BCV & LULx & - & - \\
Sakurai & 48/M & RUL to SVC & LPx & + & - \\
Sasaki & $59 / \mathrm{M}$ & RUL to SVC & RULx & - & - \\
Tanaka & $81 / \mathrm{M}$ & RUL to SVC & RULx & - & - \\
Asakura & $64 / \mathrm{M}$ & LUL to BCV & LULx & - & - \\
\hline
\end{tabular}

PAPVC: partial anomalous pulmonary venous connection; ASD: atrial septal defect; BCV: brachiocephalic vein; LLLx: left lower lobectomy; RUL: right upper lobe; LPx: left pneumonectomy; LUL: left upper lobe; LULx: left upper lobectomy; RPx: right pneumonectomy; RULx: right upper lobectomy; SVC: superior vena cava

with ligation of the anomalous vein was performed successfully. Postoperative blood gas analysis showed $\mathrm{PaO} 2$, $95 \mathrm{mmHg}$ and $\mathrm{PaCO} 2,43 \mathrm{mmHg}$ at room air. After uneventful recovery, the patient was discharged on postoperative day 11. Postoperative echocardiography revealed marginally elevated EF of $65 \%$, and reduction in pulmonary arterial pressure $(33 / 16 \mathrm{mmHg})$. He is living well without relapse of lung cancer 60 months after surgery.

\section{Discussion}

PAPVC is a relatively uncommon congenital anomaly, with a reported incidence of $0.4 \%-0.7 \% .^{1,2)}$ In previous reports focusing on children, PAPVC was commonly associated with atrial septal defects (ASD), and was found on the right side. ${ }^{3)}$ In contrast, a recent review of 29 cases of PAPVC incidentally found on CT in adults showed that $79 \%$ of PAPVC was located in the left upper lobe, and complication of ASD was very rare (3\%). ${ }^{4}$ In 7 previous case reports of PAPVC associated with lung cancer, 5 cases (63\%) had anomalous vein in left upper lobe, and none of these cases had ASD (Table 1).$^{5-11)}$ These reports indicate that incidental adult PAPVC is most frequently found in the left upper lobe, and infrequently associated with ASD.

PAPVC is distinctly visible on chest CT particularly with contrast-enhancement. It is more easily diagnosed on the left side in comparison to the right. ${ }^{4)}$ On the left side, typically, a tubular structure is detected beside aortic arch in a location where only mediastinal fat normally exists. On the other hand, PAPVC is difficult to diagnose on echocardiography or chest X-ray. In fact, although the patient in this study had been followed up by cardiologists for atrial fibrillation and mitral regurgitation, his PAPVC had not been diagnosed. In 7 previously reported cases, 5 cases were not diagnosed as having PAPVC before pulmonary resection (Table 1). In all these 5 cases, the authors showed that those PAPVCs could be detected retrospectively on preoperative chest CT.

In the present case, lung cancer was located in the same lobe as PAPVC, and lobectomy was the definitive treatment for both lung cancer and PAPVC. However, if PAPVC was located in a different lobe, major lung resection could increase the volume of shunt flow and cause right-sided heart failure. ${ }^{5)}$ In such cases, treatment for PAPVC is necessary. In previously reported 7 cases, 2 cases underwent treatment of PAPVC in associated with pulmonary resection (Table 1). In the case reported by Takei, anomalous pulmonary vein of left upper lobe was separated from the left brachiocephalic vein, and approximated with the stump of inferior pulmonary vein after left lower lobectomy. ${ }^{7)}$ In the case reported by Sakurai, anomalous pulmonary blood flow from right upper and middle lobe was flowed into superior vena cava. The anomalous blood flow had been redirected to left atrium by intra-atrial baffle rerouting under cardiopulmonary bypass, 3 weeks before left pneumonectomy was performed. ${ }^{9)}$ Surgical indication of PAPVC is a Qp/Qs ratio exceeding 1.5. ${ }^{3)}$ In present case, preoperative Qp/Qs ratio was 2.0 , and pulmonary arterial pressure decreased after lobectomy. Therefore, surgery was considered to be meritorious not only for lung cancer but also for PAPVC although the patient was asymptomatic. PAPVC is a rare congenital anomaly, but thoracic surgeons should pay attention to the presence of PAPVC on chest CT before major lung resections. 


\section{Conclusion}

We experienced a patient with PAPVC and primary lung cancer in the same lobe, which was successfully treated by lobectomy. In contrast to pediatric PAPVC, adult PAPVC are commonly associated with the left upper lobe, and are without ASD. Thoracic surgeons should pay attention to the presence of PAPVC before major lung resection because, in most cases, it is easily detectable on chest $\mathrm{CT}$ if one is aware of this condition.

\section{Disclosure Statement}

Keisuke Asakura and other co-authors have no conflict of interest.

\section{References}

1) Healey JE Jr. An anatomic survey of anomalous pulmonary veins: their clinical significance. J Thorac Surg 1952; 23: 433-44.

2) Hughes CWR, P.C. Anomalous pulmonary veins. Arch Pathol 1944; 37: 364-7.

3) AboulHosn JA, Criley JM, Stringer WW. Partial anomalous pulmonary venous return: case report and review of the literature. Catheter Cardiovasc Interv 2003; 58: $548-52$.
4) Haramati LB, Moche IE, Rivera VT, et al. Computed tomography of partial anomalous pulmonary venous connection in adults. J Comput Assist Tomogr 2003; 27: 743-9.

5) Black MD, Shamji FM, Goldstein W, et al. Pulmonary resection and contralateral anomalous venous drainage: a lethal combination. Ann Thorac Surg 1992; 53: 689-91.

6) Takamori S, Hayashi A, Nagamatsu Y, et al. Left partial anomalous pulmonary venous connection found during a lobectomy for lung cancer: report of a case. Surg Today 1995; 25: 982-3.

7) Takei H, Suzuki K, Asamura H, et al. Successful pulmonary resection of lung cancer in a patient with partial anomalous pulmonary venous connection: report of a case. Surg Today 2002; 32: 899-901.

8) Miwa K, Takamori S, Hayashi A, et al. Incidental partial anomalous pulmonary venous connection in left lung cancer. Jpn J Thorac Cardiovasc Surg 2004; 52: 189-90.

9) Sakurai H, Kondo H, Sekiguchi A, et al. Left pneumonectomy for lung cancer after correction of contralateral partial anomalous pulmonary venous return. Ann Thorac Surg 2005; 79: 1778-80.

10) Sasaki H, Naka N, Kitahara N, et al. Right partial anomalous pulmonary venous connection found during lobectomy for coexisting lung cancer and tuberculosis: report of a case. Clin Lung Cancer 2006; 7: 350-2.

11) Tanaka R, Nakazato Y, Fukura H, et al. Partial anomalous pulmonary venous connection in right lung cancer: report of a case. Surg Today. 2008; 38: 147-9. 